\title{
Expression and phosphorylation of translation regulatory protein 4E-binding protein (BP)-1 in low-risk diffuse large $B$ cell lymphoma
}

\author{
Mary J. Ninan • Ajay Rawal • Hector Mesa • \\ Dennis J. Knapp • Michael A. Kuskowski • \\ Pankaj Gupta
}

Received: 6 February 2013 / Accepted: 21 May 2013 / Published online: 8 June 2013

(C) Springer-Verlag Berlin Heidelberg (outside the USA) 2013

\begin{abstract}
Protein translation initiation is controlled by eukaryotic initiation factor 4E (eIF-4E) binding protein 1 (4E-BP1). Phosphorylated 4E-BP1 dissociates from eIF-4E, allowing translation of transcripts mediating cell cycling, survival, and angiogenesis. The expression and phosphorylation of 4E-BP1, and influence on prognosis of diffuse large B cell lymphoma (DLBCL), is unknown. Because at least some patients with low-risk (International Prognostic Index score 0-2) DLBCL treated with standard anthracycline-based chemotherapy experience a short survival, we examined if 4E-BP1 expression and phosphorylation may provide additional prognostic information in 35 patients with low-risk DLBCL. We examined their initial diagnostic pathology specimens for 4E-BP1 expression and phosphorylation using immunohistochemistry, and we
\end{abstract}

\section{J. Ninan · P. Gupta $(\bowtie)$}

Department of Medicine, Hematology-Oncology Section 111E, Minneapolis Veterans Administration Health Care System, One Veterans Drive, Minneapolis, MN 55417, USA

e-mail: gupta013@umn.edu

A. Rawal $\cdot$ H. Mesa $\cdot$ D. J. Knapp

Department of Pathology, Minneapolis Veterans Administration

Health Care System, Minneapolis, MN, USA

\section{A. Kuskowski}

Geriatric Research and Education Clinical Center (GRECC),

Minneapolis Veterans Administration Health Care System,

Minneapolis, MN, USA

M. J. Ninan · P. Gupta

Department of Medicine, Division of Hematology,

Oncology and Transplantation, University of Minnesota,

Minneapolis, MN, USA

A. Rawal $\cdot$ H. Mesa

Department of Pathology, University of Minnesota, Minneapolis, MN, USA correlated these with clinical outcomes. While 4E-BP1 was uniformly expressed, there was wide distribution in its level of phosphorylation (biological activity). 4E-BP1 phosphorylation correlated strongly with overall survival (OS; $P=0.007$ ) and progression-free survival (PFS; $P=0.02$ ) and was the most significant independent variable for both OS and PFS on multivariable analysis. Our findings suggest that immunohistochemical evaluation of 4E-BP1 phosphorylation may help refine the prognostication of patients with low-risk DLBCL. Prospective studies in an independent cohort of patients are warranted.

Keywords EIF4EBP1 protein, human · Eukaryotic initiation factor-4E · Lymphoma, large B cell, diffuse · Prognosis · Protein biosynthesis

\section{Introduction}

There has been considerable interest in identifying molecular mechanisms that contribute to the pathogenesis and clinical behavior of lymphomas in order to refine prognostication and select patients who may benefit from novel targeted therapies. Diffuse large B cell lymphoma (DLBCL) is an excellent example since it is clinically heterogeneous, and multiple efforts are ongoing to identify prognostic factors that help recognize patients who might need more aggressive or targeted first-line treatment [1].

Several cell signaling pathways have been discovered in the past decade with important clinical implications. Protein translation initiation appears to be tightly regulated by eukaryotic initiation factor 4E (eIF-4E; Fig. 1), which binds the 5' cap end of mRNA and mediates cap-dependent translation $[2,3]$. eIF-4E binding protein 1 (4E-BP1) regulates 
Fig. 1 Regulation of initiation of cap-dependent protein translation by phosphorylation status of 4E-BP1. Activation of mTOR induces phosphorylation of $4 \mathrm{E}-\mathrm{BP} 1$, resulting in release of eIF-4E in an active form. Together with eIF-4G, eIF-4A, and eIF-3, eIF-4E forms the eIF-4F translation initiation complex, which initiates capdependent translation of many proteins involved in cancer progression and metastases. Several additional signaling intermediates further influence the activity of mTOR/4E-BP1/ eIF-4E axis; these are not shown in this simplified diagram. $A$ : eukaryotic initiation factor (eIF)-4A; $A k t$ : human homolog of viral oncogene v-akt; $G$ : eIF-4G; $m R N A$, messenger RNA; $m T O R$, mammalian target of rapamycin; $P$ : phosphate; PI3-k: phosphoinositide 3-kinase; 3: eIF-3; 4E-BP1: eIF-4E binding protein 1

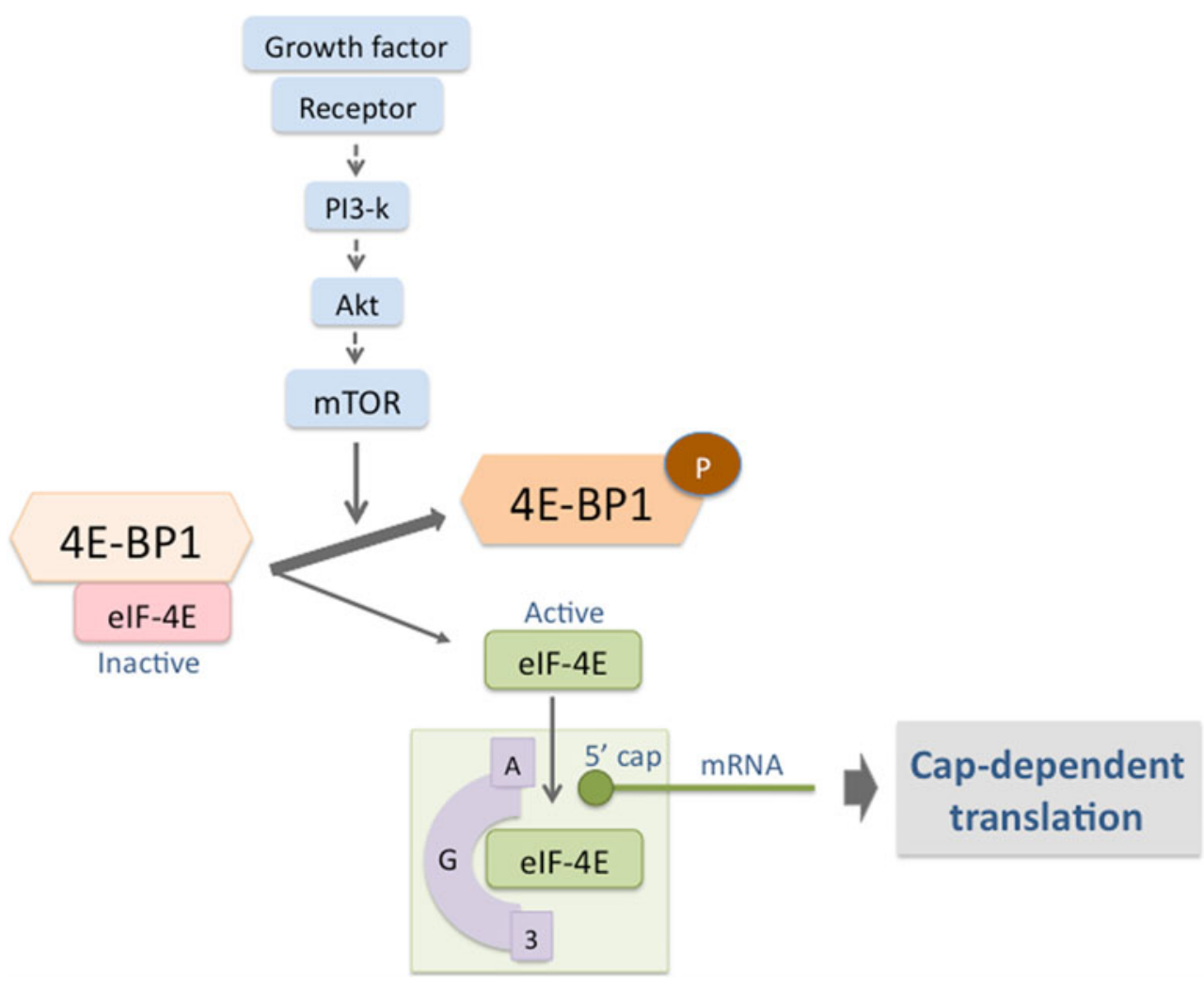

the function of eIF-4E by binding to eIF-4E in the nonphosphorylated form. Phosphorylation of 4E-BP1 (p4EBP1) dissociates the molecule from eIF-4E, thereby allowing translation of several cap-dependent transcripts responsible for cell cycling, survival, and angiogenesis, including c-myc, cyclin D1, ornithine decarboxylase, survivin, bcl-2, vascular endothelial growth factor, fibroblast growth factor 2, matrix metalloproteinase 9, and heparinase [4]. Our group reported that transfection of solid tumor cells with wild-type 4E-BP1 or the constitutively active mutant $4 \mathrm{E}-\mathrm{BP} 1^{\mathrm{A} 37 / \mathrm{A} 46}$ represses tumorigenicity and augments apoptosis following chemotherapy $[5,6]$.

eIF-4E expression itself is elevated in several carcinomas and hematopoietic malignancies, and this has been shown to correlate with disease progression and survival [7]. Furthermore,

Table 1 Clinical characteristics

\begin{tabular}{llc}
\hline & & $N(\%)$ \\
\hline Stage & I & $15(43)$ \\
& II & $12(34)$ \\
& III & $3(9)$ \\
IPI score & IV & $5(14)$ \\
& 0 & $4(11)$ \\
& 1 & $15(43)$ \\
& 2 & $16(46)$ \\
\hline
\end{tabular}

expression and phosphorylation of 4E-BP1 correlate with histological grade and clinical behavior of diverse malignancies $[4,8$, 9]. Since many signaling pathways converge on 4E-BP1, this has been termed a "funnel factor" [4], therapeutic targeting of which may be effective in many malignancies including lymphomas [10-14].

eIF-4E is involved in the pathogenesis of murine lymphoma [15] and influences the behavior of lymphomas in humans [16-20]. Less is known about the expression of its regulatory protein $4 \mathrm{E}-\mathrm{BP} 1$ in lymphomas. Others reported that 4E-BP1 is expressed in MCL [21, 22]. We recently reported that 4E-BP1 is widely expressed in most subtypes of B cell lymphomas, that the level of phosphorylation (indicative of activity) of 4E-BP1 varies widely among the different subgroups, and that in low-grade lymphomas, the

Table 2 Phosphorylation of 4E-BP1

\begin{tabular}{lc}
\hline Expression score & $N(\%)$ \\
\hline 0 & $11(31)$ \\
$1-5$ & $3(9)$ \\
$6-10$ & $2(6)$ \\
$11-15$ & $1(3)$ \\
$16-20$ & $5(14)$ \\
$21-25$ & $1(3)$ \\
$26-30$ & $12(34)$ \\
\hline
\end{tabular}


expression of phosphorylated 4E-BP1 parallels the expression of bcl-2 [23].

In the present study, we examined the expression and phosphorylation of 4E-BP1 in diagnostic samples from a cohort of patients with low-risk DLBCL and evaluated if this correlated with clinical features at diagnosis or with outcomes following first-line anthracycline-based chemotherapy.

\section{Patients and methods}

\section{Patients}

We identified 35 patients diagnosed with low-risk DLBCL (International Prognostic Index (IPI) score 0-2) at the Minneapolis VA Medical Center (MVAMC) from 1993 to 2006, for whom adequate original diagnostic tissue material and follow-up clinical data were available. Data pertaining to the diagnosis, treatment, response, and survival were obtained by retrospective chart review. The study was approved by the institutional Human Subjects Committee.

Immunohistochemical analysis

Formalin-fixed paraffin-embedded diagnostic tissues were retrieved from the archives of the Department of Pathology at the MVAMC. Tissues were stained for bcl-2 using a mouse monoclonal antibody (clone E17, Cell Marque, Rocklin, CA) at 1:50 dilution with EDTA-based antigen retrieval, total 4E-BP1 and phosphorylated 4E-BP1 (p4E-BP1 [Thr37/46]) using rabbit monoclonal anti-4E-BP1 (clone 53H11; Cell Signaling Technology, Danvers, MA) at 1:3,000 dilution and with citrate-based antigen retrieval, and rabbit monoclonal anti-p4E-BP1 (clone 236B4, Cell Signaling Technology) at 1:100 dilution and with EDTA-based antigen retrieval respectively, on a Bondmax automated immunostainer
Fig. 2 Expression of phosphorylated 4E-BP1 in diffuse large B cell lymphomas. Representative samples are shown, illustrating the spectrum of variability in expression of phosphorylated 4E-BP1 in diffuse large B cell lymphomas. All samples were stained with anti-phosphorylated 4E-BP1 as described in the text. Magnification: left panels $\times 400$, right panels $\times 1,000$. a, b: Specimen with no detectable expression in the large cells (expression score 0). c, d: Specimen with $3+$ expression in $50 \%$ of the large cells (expression score 15). e, f: Specimen with diffuse $3+$ expression in the large cells (expression score 30 )
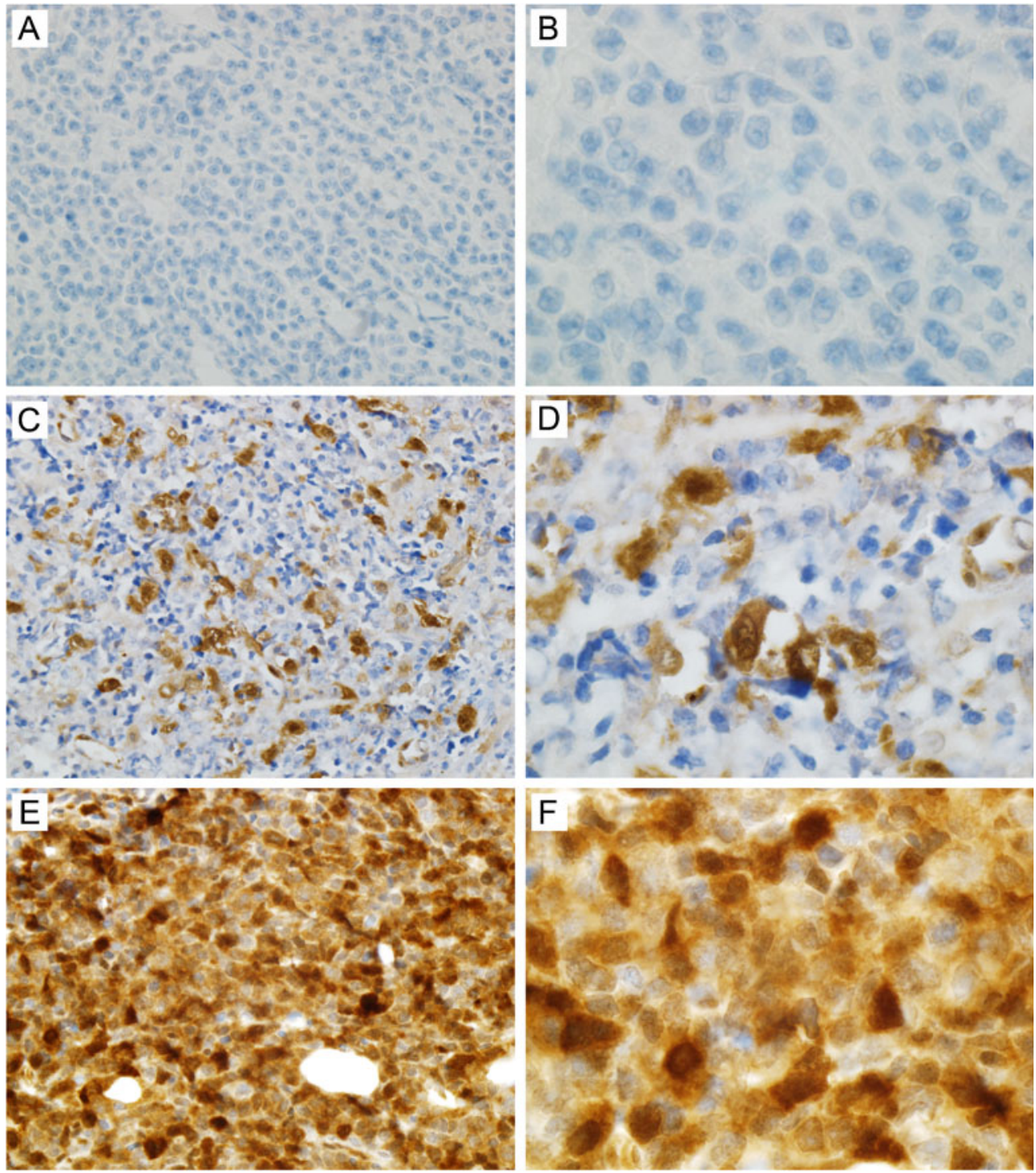
(Leica Biosystems, Bannockburn, IL) as previously described by us [23]. The slides were read by two hematopathologists (A.R. and H.M.) blinded to the clinical data and were analyzed for percentage of cells positive (scale, 0 to 100) and intensity of cytoplasmic and/or nuclear expression $(0,1+, 2+$, or $3+$, denoting none, weak, moderate, and strong staining, respectively). An expression score was calculated from their product ([\% cells positive $\times$ intensity $] / 10$ ), thus ranging from 0 to 30 , similar to previous reports [8]. A score of $0-15$ was considered to be low, and a score of 16-30 was considered to be high.

\section{Statistical analysis}

Differences between groups were compared using a two-tailed $t$ test. In patients who received chemotherapy, the probabilities of overall survival (OS) and progression-free survival (PFS) were determined using the Kaplan-Meier method. The effects of the level of phosphorylation of 4E-BP1, IPI score, and age and use of rituximab on OS and PFS were estimated using the Cox proportional hazards regression model (SPSS software, version 19.0). Survival graphs were generated using GraphPad Prism 5.0 (GraphPad Software Inc., San Diego, CA, USA).

\section{Results}

Clinical data

The median age was 68 years (range, 25-91); $71 \%$ of patients were $>60$ years. All but one of the patients were male, a reflection of the patient population at a VA hospital. Consistent with their low IPI scores, the majority (77\%) of patients had early stage (stage I or II) disease at diagnosis (Table 1). All patients had received cyclophosphamide, doxorubicin, vincristine, and prednisone (CHOP)-based chemotherapy with $(n=15)$ or without $(n=20)$ rituximab. Patients who had not received CHOP-based chemotherapy were not included in this analysis.

\section{E-BP1 expression and phosphorylation}

Most cases examined expressed total 4E-BP1 uniformly ( $97 \%$ samples; data not shown). However, there was wide distribution in the level of phosphorylation of 4E-BP1 (low [p4E-BP1 expression score 0-15] in 17 [49\%] cases and high [expression score 16-30] in 18 [51\%] cases; Table 2 and Fig. 2). Of note, phosphorylation of 4E-BP1 was undetectable (expression score 0$)$ in $11(31 \%)$ cases. All positive cases showed predominantly cytoplasmic positivity, with scattered cells additionally showing distinct nuclear positivity. The level of phosphorylation of 4E-BP1 was comparable in bcl-2positive vs bcl-2-negative samples (expression score 16.5 \pm 3.7 vs $16.7 \pm 4.6[$ mean $\pm \mathrm{SE}]$, respectively).
Association between 4E-BP1 phosphorylation and clinical outcomes

We next examined if the level of phosphorylation of 4E-BP1 varied with clinical features at diagnosis, response to chemotherapy, and survival. The level of expression or phosphorylation of 4E-BP1 did not correlate with the clinical stage or IPI score at diagnosis, and did not influence the likelihood of achieving complete remission with CHOPbased chemotherapy.

However, the level of phosphorylation of 4E-BP1 correlated strongly with overall survival (Fig. 3a). The OS was

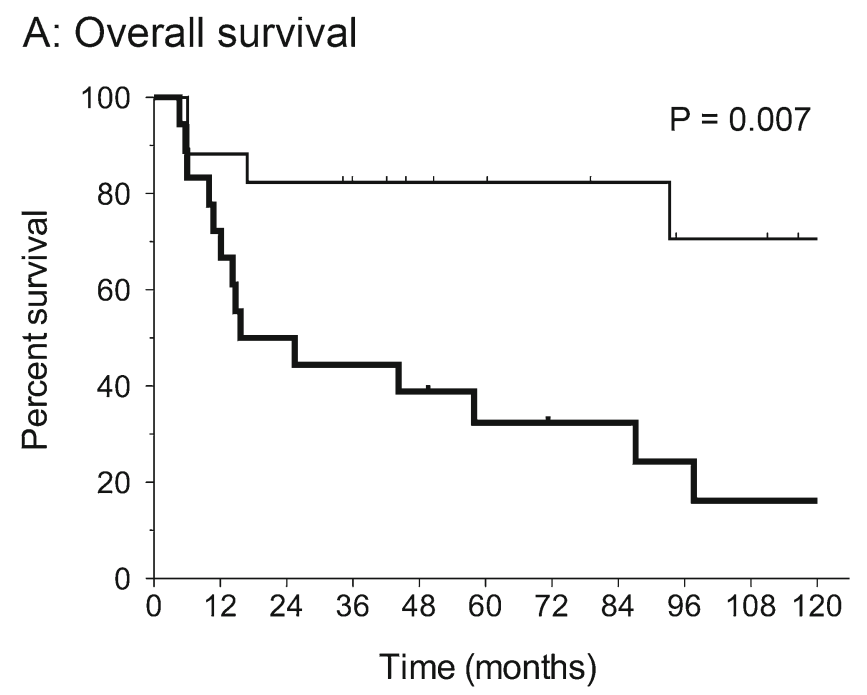

\section{B: Progression free survival}

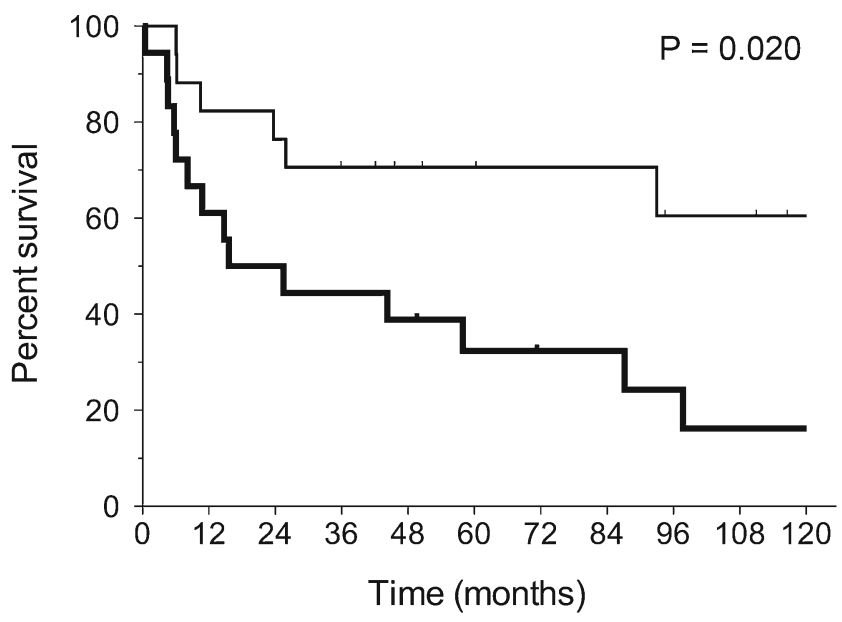

Fig. 3 Overall and progression-free survival. Higher level of phosphorylation of 4E-BP1 was associated with inferior overall survival (a) and progression-free survival (b) of clinically lower risk patients who received CHOP-based chemotherapy (IPI scores $0-2 ; N=35$ ). Low expression (thin lines): p4E-BP1 expression score $=0-15$; High expression (thick lines): p4E-BP1 expression score $=16-30$. Tick marks indicate censored cases 
significantly better $(P=0.007)$ in patients with low levels of phosphorylation of 4E-BP1 (expression score $=0-15 ; n=17$ ) compared to those with higher levels of 4E-BP1 phosphorylation (expression score $=16-30 ; n=18$ ). The median survival was 146 vs 21 months, and the 5-year survival was 82 vs $32 \%$, in patients with low and high levels of 4E-BP1 phosphorylation, respectively. Similarly, the level of phosphorylation of 4E-BP1 (low vs high) correlated significantly with progression-free survival $(P=0.02$; Fig. $3 b)$. The median PFS was 128 vs 20 months, and the 5-year survival was 71 vs $32 \%$, in patients with low and high levels of 4E-BP1 phosphorylation, respectively. The difference in survival between the two groups was not a consequence of differences in age or IPI score at diagnosis. As shown in Table 3, the level of phosphorylation of 4E-BP1 was the most impactful of these factors on both univariable and multivariable analyses for OS and PFS.

\section{Discussion}

DLBCL is a clinically heterogeneous malignancy, with marked variability in response to chemotherapy and longterm survival. Several studies have identified molecular subtypes of DLBCL with significantly different survival following anthracycline-based therapy (reviewed by Ninan et al. [1]).

The level of expression and phosphorylation of 4E-BP1 varies widely in different malignancies $[4,8,9]$. In accordance with these observations, we report that the extent of phosphorylation of 4E-BP1 varies considerably in DLBCL. A high level of expression of phosphorylated 4E-BP1 in neoplastic lymphocytes would be expected to upregulate cap-mediated translation of proteins responsible for angiogenesis, and malignant cell proliferation and survival $[4,9,10,17,18,20,24$, $25]$. The inferior survival associated with increased expression of phosphorylated 4E-BP1 that we report. DLBCL is consistent with these phenomena, and in keeping with previous reports in other malignancies including malignant melanoma and cancers of the breast, ovary, prostate and stomach [26-28]. In the present study, we limited the analysis to patients with low-risk DLBCL, in order to examine if differences in p4E-BP1 expression may help explain at least some of the known variability in outcomes of patients who are otherwise believed to have a good prognosis.

Several drugs, including ribavirin (that interferes with eIF-4E-mediated translation via interference with cap binding) and agents that inhibit the mammalian target of rapamycin (mTOR) pathway upstream of eIF-4E are already available for clinical use and have shown some therapeutic activity in myeloid and lymphoid malignancies $[12,13]$. In addition, specific antisense oligonucleotides [10] and small molecules [29] that interfere with the interaction of eIF-4E and the cap structure and thereby interrupt translation [30] are under development and in early clinical trials. The investigational agent enzastaurin (in phase III trials in DLBCL) inhibits PKC and PI3K/AKT signaling upstream of the eIF-4E pathway, inducing apoptosis of malignant cells via hypophosphorylation of 4E-BP1 [31].

Our finding that the level of phosphorylation of 4E-BP1 provides independent prognostic information in low-risk DLBCL indicates that there is a need for confirmation in a larger, prospective study where all patients are treated with R-CHOP chemotherapy. Whether the level of phosphorylation of 4E-BP1 influences the prognosis of patients with high risk DLBCL also needs to be examined prospectively. Immunohistochemical assessment of phosphorylation of 4E-BP1 might then advance to clinical use in helping to refine the prognostication of a subset of patients diagnosed with DLBCL. Furthermore, if the influence of phosphorylated 4E-BP1 on the clinical behavior of DLBCL is confirmed, it will provide the rationale for trials to evaluate if incorporation of targeted agents that inhibit the eIF-4E pathway into therapeutic regimens might improve the survival of DLBCL patients, particularly those with lower IPI scores and high levels of 4E-BP1 phosphorylation.

Table 3 Overall and progression-free survival

\begin{tabular}{|c|c|c|c|c|c|c|c|c|}
\hline & \multicolumn{4}{|l|}{ Overall survival } & \multicolumn{4}{|c|}{ Progression-free survival } \\
\hline & \multicolumn{2}{|l|}{ Univariable analysis } & \multicolumn{2}{|c|}{ Multivariable analysis } & \multicolumn{2}{|c|}{ Univariable analysis } & \multicolumn{2}{|c|}{ Multivariable analysis } \\
\hline & $\begin{array}{l}\text { Relative risk } \\
(95 \% \mathrm{CI})\end{array}$ & $P$ value & $\begin{array}{l}\text { Relative risk } \\
(95 \% \mathrm{CI})\end{array}$ & $P$ value & $\begin{array}{l}\text { Relative risk } \\
(95 \% \mathrm{CI})\end{array}$ & $P$ value & $\begin{array}{l}\text { Relative risk } \\
(95 \% \mathrm{CI})\end{array}$ & $P$ value \\
\hline $\begin{array}{l}\text { Phospho 4E-BP1 } \\
\text { (0-15 vs } 16-30)\end{array}$ & $4.68(1.53,14.29)$ & 0.007 & $3.87(1.24,12.07)$ & 0.020 & $3.12(1.19,8.14)$ & 0.020 & $2.60(0.98,6.94)$ & 0.055 \\
\hline IPI score $(0,1,2)$ & $2.58(1.16,5.73)$ & 0.020 & $2.01(0.85,4.76)$ & 0.111 & $2.26(1.09,4.69)$ & 0.029 & $1.88(0.85,4.16)$ & 0.122 \\
\hline Age (years) & $1.04(1.00,1.08)$ & 0.041 & $1.03(0.98,1.07)$ & 0.293 & $1.04(0.99,1.07)$ & 0.056 & $1.02(0.98,1.06)$ & 0.349 \\
\hline $\begin{array}{l}\text { Rituximab in regimen } \\
\text { (no vs yes) }\end{array}$ & $0.57(0.20,1.63)$ & 0.298 & $0.60(0.20,1.80)$ & 0.364 & $0.62(0.24,1.64)$ & 0.336 & $0.67(0.24,1.82)$ & 0.426 \\
\hline
\end{tabular}


Acknowledgments We thank Gloria A. Niehans, MD (Department of Pathology, VA Medical Center, Minneapolis, MN, USA) for providing archival samples for this study. This study was supported by the Veterans Health Administration.

Conflict of interest All authors have no conflict of interest.

\section{References}

1. Ninan M, Wadhwa PD, Gupta P (2011) Prognostication of diffuse large B cell lymphoma in the rituximab era. Leuk Lymphoma 52(3):360-373

2. Culjkovic B, Topisirovic I, Borden KL (2007) Controlling gene expression through RNA regulons: the role of the eukaryotic translation initiation factor eIF4E. Cell Cycle 6(1):65-69

3. Merrick WC (2004) Cap-dependent and cap-independent translation in eukaryotic systems. Gene 332:1-11

4. Armengol G, Rojo F, Castellvi J, Iglesias C, Cuatrecasas M, Pons $\mathrm{B}$ et al (2007) 4E-binding protein 1: a key molecular "funnel factor" in human cancer with clinical implications. Cancer Res 67(16):7551-7555

5. Jacobson BA, Alter MD, Kratzke MG, Frizelle SP, Zhang Y, Peterson MS et al (2006) Repression of cap-dependent translation attenuates the transformed phenotype in non-small cell lung cancer both in vitro and in vivo. Cancer Res 66(8):4256-4262

6. Jacobson BA, De A, Kratzke MG, Patel MR, Jay-Dixon J, Whitson BA et al (2009) Activated 4E-BP1 represses tumourigenesis and IGF-I-mediated activation of the eIF4F complex in mesothelioma. Br J Cancer 101(3):424-431

7. De Benedetti A, Graff JR (2004) eIF-4E expression and its role in malignancies and metastases. Oncogene 23(18):3189-3199

8. Graff JR, Konicek BW, Lynch RL, Dumstorf CA, Dowless MS, McNulty AM et al (2009) eIF4E activation is commonly elevated in advanced human prostate cancers and significantly related to reduced patient survival. Cancer Res 69(9):3866-3873

9. Thumma SC, Kratzke RA (2007) Translational control: a target for cancer therapy. Cancer Lett 258(1):1-8

10. Graff JR, Konicek BW, Carter JH, Marcusson EG (2008) Targeting the eukaryotic translation initiation factor 4E for cancer therapy. Cancer Res 68(3):631-634

11. Graff JR, Konicek BW, Vincent TM, Lynch RL, Monteith D, Weir $\mathrm{SN}$ et al (2007) Therapeutic suppression of translation initiation factor eIF4E expression reduces tumor growth without toxicity. J Clin Invest 117(9):2638-2648

12. Assouline S, Culjkovic B, Cocolakis E, Rousseau C, Beslu N, Amri A et al (2009) Molecular targeting of the oncogene eIF4E in acute myeloid leukemia (AML): a proof-of-principle clinical trial with ribavirin. Blood 114(2):257-260

13. Rizzieri DA, Feldman E, Dipersio JF, Gabrail N, Stock W, Strair R et al (2008) A phase 2 clinical trial of deforolimus (AP23573, MK8669), a novel mammalian target of rapamycin inhibitor, in patients with relapsed or refractory hematologic malignancies. Clin Cancer Res 14(9):2756-2762

14. Bordeleau ME, Robert F, Gerard B, Lindqvist L, Chen SM, Wendel HG et al (2008) Therapeutic suppression of translation initiation modulates chemosensitivity in a mouse lymphoma model. J Clin Invest 118(7):2651-2660

15. Ruggero D, Montanaro L, Ma L, Xu W, Londei P, Cordon-Cardo C et al (2004) The translation factor eIF-4E promotes tumor formation and cooperates with c-Myc in lymphomagenesis. Nat Med 10(5):484-486

16. Wang S, Rosenwald IB, Hutzler MJ, Pihan GA, Savas L, Chen JJ et al (1999) Expression of the eukaryotic translation initiation factors 4E and 2alpha in non-Hodgkin's lymphomas. Am J Pathol 155(1):247-255

17. Wendel HG, Silva RL, Malina A, Mills JR, Zhu H, Ueda T et al (2007) Dissecting eIF4E action in tumorigenesis. Genes Dev 21(24):3232-3237

18. Yang SX, Hewitt SM, Steinberg SM, Liewehr DJ, Swain SM (2007) Expression levels of eIF4E, VEGF, and cyclin D1, and correlation of eIF4E with VEGF and cyclin D1 in multi-tumor tissue microarray. Oncol Rep 17(2):281-287

19. Zhao Y, Liu W, Zhou S, Zhou J, Sun H (2005) Relationship between eukaryotic translation initiation factor $4 \mathrm{E}$ and malignant angiogenesis in non-Hodgkin lymphoma. J Huazhong Univ Sci Technol Med Sci 25(6):636-638

20. Wendel HG, De Stanchina E, Fridman JS, Malina A, Ray S, Kogan $S$ et al (2004) Survival signalling by Akt and eIF4E in oncogenesis and cancer therapy. Nature 428(6980):332-337

21. Peponi E, Drakos E, Reyes G, Leventaki V, Rassidakis GZ, Medeiros LJ (2006) Activation of mammalian target of rapamycin signaling promotes cell cycle progression and protects cells from apoptosis in mantle cell lymphoma. Am J Pathol 169(6):21712180

22. Kawamata N, Chen J, Koeffler HP (2007) Suberoylanilide hydroxamic acid (SAHA; vorinostat) suppresses translation of cyclin D1 in mantle cell lymphoma cells. Blood 110(7):2667-2673

23. Kodali D, Rawal A, Ninan M, Patel MR, Mesa H, Knapp D et al (2011) Expression and phosphorylation of eukaryotic translation initiation factor $4 \mathrm{E}$ binding protein 1 in B-cell lymphomas and reactive lymphoid tissues. Arch Pathol Lab Med 135(3):365-371

24. Graff JR, Zimmer SG (2003) Translational control and metastatic progression: enhanced activity of the mRNA cap-binding protein eIF-4E selectively enhances translation of metastasis-related mRNAs. Clin Exp Metastasis 20(3):265-273

25. Ma XM, Blenis J (2009) Molecular mechanisms of mTORmediated translational control. Nat Rev Mol Cell Biol 10(5):307318

26. Castellvi J, Garcia A, Rojo F, Ruiz-Marcellan C, Gil A, Baselga J et al (2006) Phosphorylated 4E binding protein 1: a hallmark of cell signaling that correlates with survival in ovarian cancer. Cancer 107(8):1801-1811

27. O'Reilly KE, Warycha M, Davies MA, Rodrik V, Zhou XK, Yee H et al (2009) Phosphorylated 4E-BP1 is associated with poor survival in melanoma. Clin Cancer Res 15(8):2872-2878

28. Rojo F, Najera L, Lirola J, Jimenez J, Guzman M, Sabadell MD et al (2007) 4E-binding protein 1, a cell signaling hallmark in breast cancer that correlates with pathologic grade and prognosis. Clin Cancer Res 13(1):81-89

29. Moerke NJ, Aktas H, Chen H, Cantel S, Reibarkh MY, Fahmy A et al (2007) Small-molecule inhibition of the interaction between the translation initiation factors eIF4E and eIF4G. Cell 128(2):257267

30. Konicek BW, Dumstorf CA, Graff JR (2008) Targeting the eIF4F translation initiation complex for cancer therapy. Cell Cycle 7(16):2466-2471

31. Dumstorf CA, Konicek BW, McNulty AM, Parsons SH, Furic L, Sonenberg N et al (2010) Modulation of 4E-BP1 function as a critical determinant of enzastaurin-induced apoptosis. Mol Cancer Ther 9(12):3158-3163 